\section{SAT0239 PREDICTION OF LONG-TERM EVOLUTIONARY PROFILES IN EOSINOPHILIC GRANULOMATOSIS WITH POLYANGIITIS (CHURG-STRAUSS) BASED ON BASELINE AND FOLLOW-UP CHARACTERISTICS}

Matthias Papo ${ }^{1}$, Renato A. Sinico ${ }^{2}$, Vítor Teixeira ${ }^{3}$, Maria-Letizia Urban ${ }^{4}$ Juliane Mahrhold ${ }^{5}$, Sara Monti ${ }^{6}$, Giulia Cassone ${ }^{7}$, Franco Schiavon ${ }^{8}$, Benjamin Seeliger ${ }^{9}$, Thomas Neumann ${ }^{10}$, Claus Kroegel ${ }^{11}$, Matthieu Groh ${ }^{12}$ Chiara Marvisi ${ }^{13}$, Maxime Samson ${ }^{14}$, Thomas Barba ${ }^{15}$, David Jayne ${ }^{3}$, Bernhard Hellmich ${ }^{5}$, Carlomaurizio Montecucco ${ }^{6}$, Carlo Salvarani ${ }^{7}$, JeanEmmanuel Kahn ${ }^{12}$, Bernard Bonnotte ${ }^{14}$, Cécile-Audrey Durel ${ }^{15}$, Luc Mouthon ${ }^{1}$, Xavier Puéchal ${ }^{1}$, Loïc Guillevin ${ }^{1}$, Giacomo Emmi ${ }^{4}$, Augusto Vaglio ${ }^{13}$, Benjamin Terrier', French Vasculitis Study Group and EGPA European Collaborative Initiative. ${ }^{1}$ Internal Medicine, Paris, France; ${ }^{2}$ Medicine and Surgery, Milan, Italy, ${ }^{3}$ Medicine, Cambridge, United Kingdom; ${ }^{4}$ Experimental and Clinical Medicine, Florence, Italy; Internal Medicine, Kirchheim, Germany; ${ }^{6}$ Rheumatology, Pavia, Italy, ${ }^{7}$ Rheumatology, Reggio Emilia and Modena, Italy; ${ }^{8}$ Rheumatology, Padova, Italy; ${ }^{9}$ Respiratory Medicine, Hannover, Germany; ${ }^{10}$ Rheumatology, St. Gallen, Switzerland; ${ }^{11}$ Pneumology, Jena, Germany, ${ }^{12}$ Internal Medicine, Suresnes, France; ${ }^{13}$ Nephrology, Parma, Italy, ${ }^{14}$ Internal Medicine, Dijon, France; ${ }^{15}$ Internal Medicine, Lyon, France

Background: Eosinophilic granulomatosis with polyangiitis (EGPA) (ChurgStrauss) is a small-vessel necrotizing vasculitis characterized by blood and tissue eosinophilia and asthma. Glucocorticoids (GCs) control the disease, but GC-dependence is frequent. Evolving concepts distinguish vasculitis-related symptoms from asthma and/or ENT manifestations. That distinction has become even more important since the development of Bcell and eosinophil-targeted therapies.

Objectives: This study aimed to describe and identify characteristics predicting long-term EGPA outcomes.

Methods: We set up a multicenter European cohort that included 636 EGPA patients. Based on recent consensus, we distinguished 4 EGPAevolutionary profiles: GC-dependent asthma and/or ENT manifestations (requiring prednisone $>7.5 \mathrm{mg} / \mathrm{d}$ ), $\geq 1$ vasculitis relapse(s) (excluding asthma and/or ENT flares), both phenotypes, and complete remission (no GC-dependent asthma/ENT signs, no vasculitis relapse and prednisone $<5 \mathrm{mg} / \mathrm{d}$ at last follow-up). Baseline and follow-up characteristics predicting those outcomes were analyzed.

Results: After median follow-up of 63 (IQR 30-110) months, 35.8\% had GC-dependent asthma and/or ENT manifestations, $12.9 \%$ had $\geq 1$ vasculitis relapse(s), $14.3 \%$ had both phenotypes, $14.6 \%$ were in complete remission, $14.4 \%$ were in partial remission and $7.8 \%$ had not reach remission.

Patients with GC-dependent asthma/ENT manifestations were younger at diagnosis $(p<0.0001)$, had more frequent GC-treated asthma before overt EGPA $(p=0.002)$, had more ENT manifestations $(p=0.01)$ and less frequent MPO-ANCA $(p<0.0001)$. Their daily GC dose was higher at every time point $(p<0.0001)$, and they had more frequent active asthma at last follow-up $(p<0.0001)$.

Patients with vasculitis relapse(s) had more frequently neurological manifestations at diagnosis $(p=0.002)$ and MPO-ANCA positivity $(p<0.0001)$, and less frequently pulmonary infiltrates $(p=0.031)$. Median time from diagnosis-to- $1^{\text {st }}$ vasculitis relapse was $25(11-60)$ months. During follow-up, their daily GC dose was lower than those with GC-dependent asthma/ ENT manifestations, but similar to those in complete remission. At last follow-up, neurological sequelae tended to be more frequent $(p=0.06)$.

Finally, patients in complete remission were older $(p<0.0001)$, had more fever $(p=0.03)$, less GC-treated asthma $(p=0.002)$ and ENT manifestations at diagnosis $(p=0.01)$, lower daily $\mathrm{GC}$ dose during follow-up $(p<0.0001)$, lower eosinophils count at 6 months $(p=0.002)$ and less frequent sequelae $(p=0.003)$

Conclusion: EGPA seems to evolve toward distinct phenotypic profiles, which could be identified using baseline and follow-up characteristics. Early identification of those profiles could allow guided choices of the best therapeutic option.

Disclosure of Interests: Matthias Papo: None declared, Renato A. Sinico: None declared, Vítor Teixeira: None declared, Maria-Letizia Urban: None declared, Juliane Mahrhold: None declared, Sara Monti: None declared, Giulia Cassone: None declared, Franco Schiavon: None declared, Benjamin Seeliger: None declared, Thomas Neumann: None declared, Claus Kroegel: None declared, Matthieu Groh: None declared, Chiara Marvisi: None declared, Maxime Samson: None declared, Thomas Barba: None declared, David Jayne Grant/research support from: David Jayne has received research grants from Chemocentryx, GSK, Roche/Genentech and Sanofi-Genzyme. He has received consultancy fees from Astra-Zeneca, Boehringer-Ingelheim, Chemocentryx, Chugai, GSK, Infla-RX, Insmed and Takeda, Bernhard Hellmich Consultant for: Roche, Speakers bureau: Abbvie, MSD, Roche, Novartis, Pfizer, Carlomaurizio Montecucco Speakers bureau: AbbVie, Bristol-Myers Squibb, Celgene, Sanofi, Genzyme, Lilly, MSD, Pfizer, UCB, Carlo Salvarani Grant/research support from: Roche, Consultant for: Eli Lilly and Company, Roche, Abbvie, Jean-Emmanue Kahn: None declared, Bernard Bonnotte: None declared, Cécile-Audrey Durel: None declared, Luc Mouthon: None declared, Xavier Puéchal: None declared, Loïc Guillevin: None declared, Giacomo Emmi: None declared, Augusto Vaglio: None declared, Benjamin Terrier: None declared

DOI: 10.1136/annrheumdis-2019-eular.4013

\section{SAT0240 DEVELOPMENT AND PRELIMINARY VALIDATION OF THE BEHCYET'S SYNDROME OVERALL DAMAGE INDEX (BODI)}

Matteo Piga ${ }^{1}$, Alberto Floris ${ }^{1}$, Gerard Espinosa ${ }^{2}$, Nikolaos Kougkas ${ }^{3}$, Andrea Lo Monaco $^{4}$, Giuseppe Lopalco ${ }^{5}$, Ida Orlando ${ }^{6}$, Vittorio Pirani ${ }^{\text {, }}$

Ernestina Santos ${ }^{8,9}$, Luísa Serpa Pinto ${ }^{10}$, George Bertsias ${ }^{3}$, Luca Cantarini ${ }^{6}$, Alberto Cauli ${ }^{1}$, Ricard Cervera ${ }^{2}$, João Correia ${ }^{10}$, Marcello Govoni ${ }^{4}$, Florenzo lannone ${ }^{5}$, Ana Martins Da Silva 8,9 , Piergiorgio Neri ${ }^{11}$

Carlos Vasconcelos ${ }^{9}$, Monica Muntoni ${ }^{12}$, Alessandro Mathieu ${ }^{1} .{ }^{1}$ University of Cagliari, Rheumatology, Monserrato, Italy, ${ }^{2}$ University of Barcelona, Autoimmune Diseases, Barcelona, Catalonia, Spain; ${ }^{3}$ University of Crete, Rheumatology, Clinical Immunology and Allergy, Heraklion, Greece; ${ }^{4}$ University of Ferrara, Rheumatology, Ferrara, Italy; ${ }^{5}$ University of Bari, Rheumatology, Bari, Italy; ${ }^{6}$ University of Siena, Rheumatology, Siena, Italy; ${ }^{7}$ Università Politecnica delle Marche, Ophthalmology, Ancona, Italy, ${ }^{8}$ Centro Hospitalar do Porto/Hospital de Santo António, Neurology, Porto, Portugal; ${ }^{9}$ University of Porto, UMIB, Abel Salazar Biomedical Sciences Institute, Porto, Portugal; ${ }^{10}$ Hospital Santo Antonio Centro Hospitalar do Porto, Unidade de Imunologia Clinica, Porto, Portugal;

${ }^{11}$ Cleveland Clinic Abu Dhabi, Eye Institute, Abu Dhabi, United Arab Emirates;

${ }^{12}$ Patient delegate, Cagliari, Italy

Background: Irreversible organ damage is considered a core outcome by the OMERACT working group. However, no specific tools are currently available to detect and measure damage accrual in Behçet's syndrome (BS).

Objectives: To develop and preliminarily validate the Behçet's syndrome Overall Damage Index (BODI). NCT03803462.

Methods: A preliminary version of the instrument (p-BODI) was developed by reviewing pre-existing tools [e.g. Vasculitis damage index (VDI)] and through an extensive literature review. p-BODI was then reviewed and implemented by a multi-rounds Delphi process, involving an international and multidisciplinary (5 rheumatologists, 4 internist, 1 ophthalmologist, 1 neurologist) panel of experts in BS management and a patients' delegate. A group of clinicians (CG), not involved in the BODI development, was asked to independently score a set of clinical vignettes, in order to test the instrument reliability, after a training process consisting of a user manual and a video-tutorial. Then, Cohen's $K$ and Intra-class correlation coefficient (ICC) between assessors and gold standard were calculated. Afterwards, BODI validation was conducted according to the OMERACT Filter 2.0 in a multicenter BS cohort.

Results: Starting from a list of 120 candidate items, the final version of BODI consisted of 4 overarching principles, 30 items and 12 sub-items (each of them scores one point) grouped in 8 domains (figure).

In terms of reliability, the mean $\mathrm{K}$ coefficient was $0.84(95 \% \mathrm{Cl} 0.78$ to $0.90)$ and the ICC was $0.88(95 \% \mathrm{Cl} 0.80-0.95)$.

Validation cohort consisted of 228 BS patients $(49.1 \%$ males), with a median (IQR) age and disease duration of 46.9 (35.5-55.0) and 11.7 (5.8-20.7) years, respectively. Overall, prevalence of any BODI damage (BODI $\geq 1$ ) was $56.1 \%$ with a median score of $1.0(0-2.0)$. In regard of construct validity, BODI score significantly correlated with VDI (Spearman's rho $0.693, p<0.001)$. Besides, BODI score did not correlate with BDCAF (rho-0.016, $\mathrm{p}=0.807$ ), contrary to VDI (rho $0.141, \mathrm{p}=0.034$ ). Such results support the validity of $\mathrm{BODI}$, unlike $\mathrm{VDI}$, in discriminating damage from current disease activity in BS. On multiple regression analysis, factors independently associated to higher BODI damage score were male gender $(\beta$ coefficient $0.143 ; p=0.014)$, longer disease duration $(\beta 0.221 ; p<$ $0.001)$, past major organ involvement $(\beta 0.377 ; p<0.001)$ and required use of anti-TNF $\alpha$ inhibitors $(\beta$ 0.222; $p<0.001$ ).

Full agreement among the $C G$ was reached in judging BODI as a credible, comprehensive, easy to use, timesaving and acceptable instrument. Conclusion: BODI is the first tool specifically developed to assess damage in BS. Preliminary data encourage further validation of BODI in more extended and multi-ethnic BS cohorts before being applied in clinical practice and as a therapeutic outcome. 




Disclosure of Interests: Matteo Piga: None declared, Alberto Floris: None declared, Gerard Espinosa: None declared, Nikolaos Kougkas: None declared, Andrea Lo Monaco: None declared, Giuseppe Lopalco Speakers bureau: SOBI, BMS, Ida Orlando: None declared, Vittorio Pirani: None declared, Ernestina Santos: None declared, Luísa Serpa Pinto: None declared, George Bertsias: None declared, Luca Cantarini: None declared, Alberto Cauli: None declared, Ricard Cervera: None declared, João Correia: None declared, Marcello Govoni: None declared, Florenzo lannone Consultant for: $\mathrm{F}$ lannone has received consultancy fees and/or speaker honoraria from Pfizer, AbbVie, MSD, BMS, Novartis, Lilly, UCB outside this work, Speakers bureau: F lannone has received consultancy fees and/or speaker honoraria from Pfizer, AbbVie, MSD, BMS, Novartis, Lilly, UCB outside this work, Ana Martins da Silva: None declared, Piergiorgio Neri: None declared, Carlos Vasconcelos: None declared, Monica Muntoni: None declared, Alessandro Mathieu: None declared

DOI: 10.1136/annrheumdis-2019-eular.3517

\section{SAT0241 HEALED TEMPORAL ARTERITIS. ANALYSIS OF PATIENTS REFERRED TO RHEUMATOLOGY WITH A DIAGNOSIS OF TEMPORAL ARTERITIS}

Lily Romero Karam ${ }^{1}$, Shruti Agashe ${ }^{2}$, lleana De Anda-Duran ${ }^{3}$, Ramona Mihu ${ }^{4}$, Jovan Popovich ${ }^{4} .{ }^{1}$ Houston Methodist, Internal Medicine, Houston, United States of America; ${ }^{2}$ Houston Methodist, Neurology, Houston, United States of America; ${ }^{3}$ Harvard T.H. Chan School of Public Health, MPH-Epidemiology, Boston, United States of America; ${ }^{4}$ Houston Methodist, Rheumatology, Houston, United States of America

Background: The diagnosis of giant cell arteritis (GCA), or temporal arteritis (TA), is a clinical one. Temporal artery biopsy is usually obtained to strengthen support for treatment. Unfortunately a biopsy does not always confirm the diagnosis; results can be reported as active, healed, or negative even in patients with all other features of giant cell arteritis. Histopathologic features suggestive of resolving inflammation are evident in healed TA, but these are non-specific and can also be seen in other conditions that are unlikely to benefit from prolonged steroid treatment. It is not clear if a pathological diagnosis of healed TA helps to support treatment which may be associated with significant toxicity.

Objectives: We analyzed clinical features of patients with "healed" TA and compared them to patients with active and negative biopsies with the goal of identifying any features that may aid treatment decisions.

Methods: Retrospective analysis of 49 patients with established TA diagnosis, seen at a rheumatology practice in Houston, Texas from 8/2007 to 12/2018. Fifty seven percent were referred by Ophthalmology, the rest by other specialties. Patients were divided into 3 groups based on biopsy: Active, Healed, and Negative. Clinical features analyzed at the time of presentation were gender, age of onset, fulfillment of the American College of Rheumatology (ACR) 1990 criteria for the classification of Giant Cell Arteritis and the 2016 revised ACR criteria for early diagnosis of Giant Cell Arteritis (rACR), and the presence of vision abnormalities, headache, temporal artery tenderness/induration, jaw claudication, fever, anemia, sedimentation rate at onset, and flares during follow up.

Descriptive data was reported as means \pm standard deviation for continuous variables and as proportions for categorical variables; analyzed with ANOVA and chi squared, respectively. Post hoc analysis with Bonferroni corrections and Fisher's exact tests were used. All analyses were performed on Stata version 15 (StataCorp LP, College Station, TX, USA). A p-value of $\leq 0.05$ was considered statistically significant.

Results: Thirty-two (65\%) of the 49 patients were female and 17 (35\%) were male. Biopsy reports showed active disease in 14 (28.5\%), healed in $31(63.3 \%)$ and negative in $4(8.2 \%)$ patients. Average age was 72.7 years $\pm 6.3,73.0 \pm 9.1,61.25 \pm 1.3$ years in these 3 groups, respectively ( $p$ value 0.008 ). The Active versus Healed groups were statistically different with respect to age ( $p$ 0.028), those meeting ACR and rACR criteria ( $p$ 0.029 and $p$ 0.002), and the incidence of jaw claudication: 8 active $(57.1 \%)$ versus 5 healed $(16.1 \%)$ patients ( $p$ 0.05). There was no statistically significant difference in ophthalmologic manifestations, headache, temporal artery palpation, fever, anemia, and flares. In addition, $90 \%$ of patients reported side effects from steroids.

Conclusion: The percent of healed TA was $63.3 \%$, higher than in other studies. Patients with healed TA were less likely to fulfill ACR and rACR criteria for giant cell arteritis. Further studies are needed to identify the subset of patients with healed TA who would have a clear benefit from high dose steroids, that would outweigh the treatment side effects. At the end of the day, the diagnosis of TA remains a clinical one and early involvement of Rheumatology may help avoid unnecessary biopsies and treatment. Ultrasound, MRA, and PET-CT scan may be able to fill the gap when biopsy fails to confirm the diagnosis.

Disclosure of Interests: None declared

DOI: 10.1136/annrheumdis-2019-eular.3559

\section{SAT0242 SENSITIVITY OF TEMPORAL ARTERY BIOPSY IN GIANT CELL ARTERITIS: SYSTEMATIC LITERATURE REVIEW AND META-ANALYSIS OF CLINICAL DATA}

Emma Rubenstein ${ }^{1}$, Carla Maldini ${ }^{1}$, Solange Gonzalez-Chiappe ${ }^{1}$, Sylvie Chevret $^{2}$, Alfred Mahr'. ' Saint Louis University Hospital, Department of Internal Medicine, Paris, France; ${ }^{2}$ Saint Louis University Hospital, Department of Biostatistics, Paris, France

Background: The role of temporal artery biopsy (TAB) as a reference test for the diagnosis of giant cell arteritis (GCA) is currently questioned by the use of non-invasive imaging techniques such as temporal artery ultrasonography (TA-US). Although TAB is highly specific, a subset of patients with a clinical diagnosis of GCA does not show the characteristic histopathological signs. The lack of knowledge of the proportion of GCA cases with positive findings on TAB hampers comparisons of the sensitivity of TAB and imaging tests for diagnosing GCA.

Objectives: We performed a systematic literature review and meta-analysis to estimate the sensitivity of TAB in GCA and to identify factors that may influence the estimate.

Methods: We searched MEDLINE via PubMed, EMBASE and CENTRAL databases for articles reporting $T A B$ in $G C A$ that were published from 1990 to 2017, with no language restriction. Eligibility criteria included studies with $\geq 30$ GCA cases fulfilling the original or modified 1990 ACR classification criteria for GCA. From eligible publications, two independent researchers extracted the main methodological, geographic, demographic, and clinical characteristics and the number of TAB-positive cases among all cases with interpretable results for TAB. By meta-analysis, we computed the pooled proportion of TAB-positive GCA cases by using a random-effects model with a binomial-normal distribution and assessed heterogeneity by the $\mathrm{I}^{2}$ statistic. Subgroup and meta-regression analyses were used to examine the effect of 16 covariates (e.g., geographic, demographic, clinical and study descriptors) on TAB positivity.

Results: Among 3820 screened publications, 32 independent studies (3092 GCA patients in total) were used for the analysis. The pooled proportion of TAB positivity was estimated at $77.3 \%(95 \%$ confidence interval $71.8-81.9 \%)$, with high between-study heterogeneity $\left(F^{2}=90 \%\right)$. Subgroup analysis suggested a potential influence of year of publication (Table). This result was confirmed by univariate $(P=0.0008)$ and multivariate metaregression $(P=0.0004)$. No other analyzed covariate significantly influenced the sensitivity of TAB in GCA.

Conclusion: The $77 \%$ estimated sensitivity of TAB in GCA indicates that it is not inferior to that of TA-US (1). The decline in TAB-positive GCA cases over time could reflect an increasing propensity of clinicians to accept GCA diagnosis in the absence of proof by TAB. The unexplained high between-study heterogeneity could also result from differences in $\mathrm{TAB}$ sampling, processing or interpretation.

\section{REFERENCE}

[1] Duftner C, Dejaco C, Sepriano A, et al. Imaging in diagnosis, outcome prediction and monitoring of large vessel vasculitis: a systematic literature review and meta-analysis informing the EULAR recommendations. RMD Open. 2018;4:e000612. 